\title{
Optimization of growth processes of the selected strain Lactobacillus acidophilus (B- 4107) K-1-T with the prospect of its use for the prevention of gastrointestinal diseases in calves
}

\author{
Viktor Samoylenko ${ }^{1 *}$, Nadezhda Ozheredova ${ }^{1}$,Elena Svetlakova $^{1}$, Danil Ranyuk ${ }^{1}$, and \\ Roman Ranyuk ${ }^{1}$
}

${ }^{1}$ Stavropol State Agrarian University, 12, Zootekhnicheskiy lane, 355017, Stavropol, Russia

\begin{abstract}
Gastrointestinal diseases of young cattle occupy the bulk of the total number of postnatally significant diseases in terms of coverage, which causes significant economic damage to animal husbandry. In the modern world, the basis for prophylactic that is safe for a living organism is the use of bacterial preparations created from symbiotic selected probiotic strains with the inclusion of a functional substance and a prebiotic. In this regard, the role of lactulose, which meets all the necessary international requirements, becomes particularly important. When determining the influence of the prebiotic Lactulose, as a catalytic enzyme, on the growth processes of the collection strain Lactobacillus acidophilus (B-4107) K-1$\mathrm{T}$, it was experimentally established that the prebiotic. Lactulose has a significant effect on the growth and activity of the tested strain of lactic acid microorganisms. The following data is necessary for the development of effective means of normalizing the microbiota in the prophylactic of diseases of the gastrointestinal tract of young animals of infectious and non-infectious etiology.
\end{abstract}

\section{Introduction}

Numerous studies have shown that the intestinal microflora and its metabolic activity have a significant impact on the health and productivity of ruminants. Gastrointestinal diseases of cattle occupy the main part of the total number of nosocomial diseases in terms of coverage, which causes significant economic damage to animal husbandry. The concept of prophylaxis maintained through antibiotic therapy leads to significant environmental problems, since the antibiotic-resistant properties of bacteria in the body of animals, in particular, in the large intestine, continue to grow steadily, resulting in a decrease in the effectiveness of the method used $[6,13]$.

The use of antibiotic prophylaxis for young cattle during the colostrum or neonatal period (7-10 days), the milk period (up to 2 months) may become especially critical, since

* Corresponding author: viktor_samoylenko_26@mail.ru 
at this moment in the calf's life the formation (maturation) of immunocompetent organs and mechanisms of the immune response occurs for antigens, and antibiotics are known to have immunosuppressive functions [1, 14]. In the development of breeding, special attention should be paid to the choice of measures for the prevention and control of gastrointestinal diseases of young cattle in early postnatal ontogenesis [4]. These circumstances, in turn, led to practical research in the field of complex use of bacteria-containing preparations and products-probiotics, for the correction of the intestinal microbiota of animals, as well as in order to prevent the occurrence of dysbiosis and increase the adaptive properties of the newborn to adverse environmental factors [7].

The first definition of the term "probiotic" was given in 2001 by Food and Agriculture Organization/World Health Organization. Detailed research in the field of this topic continues in the modern world of science. In 2014, the concepts of "prebiotics" and "probiotics" studied by the scientists of the International Scientific Association for Probiotics and Prebiotics (ISAPP) have undergone adjustments and now it is believed that in order to provide a therapeutic and prophylactic effect, live microorganisms called probiotics are used in the required amount on the animal's body. It is taken into account that probiotics and prebiotics have a general therapeutic and prophylactic effect on the digestive system, which is confirmed by scientists from various countries who conducted microbiological studies using strains of Lactobacillus and Bifidobacterium cultures [16, 2].

In all parts of the gastrointestinal tract, bacteria of the genus Lactobacillus persist, which have morphological properties: gram-positive obligate or facultative anaerobes. Lactobacilli, in the large intestine, live in the amount of $10^{7}-10^{8} \mathrm{CFU} / \mathrm{g}$ [5]. The metabolic function of the studied bacteria is the production of biologically active antibacterial substances with their participation in the processes of lipolysis and protealysis. In addition, during the metabolism of lactose, lactobacilli produce enzymes such as glycolase, galactosidase, and lactic dehydrogenases. When stimulating the phagocytic activity of neutrophils and macrophages, lactobacilli play the most important role, synthesizing immunoglobulins, in parallel, cellular immunity is activated and the production of immunoglobulin E stops and the immunomodulatory functions of the body are stimulated $[3,2,15]$.

It has been established that the most powerful antagonist to potentially-pathogenic and pathogenic microflora is currently Lactobacillus acidophilus. Back in 1900, Aime Morot described a microbe related to acidophilus bacilli, which he isolated from the stool of an infant. It was later renamed Bacillus acidophilus, it is now called Lactobacillus acidophilus [5].

In the production of pharmaceutical and veterinary drugs, therapeutic and prophylactic food and feed additives, selective strains of the above microorganism are used as a basis. All drugs made from these strains have probiotic properties, which, as noted earlier, have antagonistic activity against pathogenic, potentially pathogenic and putrefactive microflora $[6,12]$.

In this case, the basis of prevention is the use of bacterial medication created from symbiotic selected strains with the inclusion of a functional substance, which prevent the formation of toxic products of protein breakdown, reducing the load on the liver and kidneys, a separate and most important thing is to stimulate the growth of beneficial flora.

In this regard, the role of lactulose (prebiotic No. 1 in the world) becomes particularly important. Its use in clinical conditions as a laxative has long been included in the practice of medicine, but with a significant reduction in dosage, lactulose has a bifidogenic effect, is fermented by beneficial bacteria from the genus Lactobacillus, inducing beneficial intestinal flora, thereby altering the final microbial products $[8,9]$.

Lactulose is a synthetic disaccharide, produced by a chemical reaction from lactose, consists of two disaccharide molecules connected by a b-glycosidic bond, which 
contributes to its undeniable competitiveness in the world prebiotic market. The Food and Agriculture Organization of the United Nations has designated Lactulose as a prebiotic. The positive effect of lactulose on the gastrointestinal microflora of humans, pigs and rats has already been reported. Guerra-Ordaz noted the positive growth dynamics of newborn piglets, which were given a $1 \%$ nutritional supplement of lactulose, and this method was also marked by an increase in feed intake (FI) accompanied by integral intestinal protection [10].

In addition, during the fermentation of lactulose, a significant increase in acetic acid occurs, and the concentration of lactic, propionic and butyric acids is accompanied by a slight increase, due to which an increase in acidity occurs, in turn this leads to the suppression of putrefactive microflora, reduces the toxic load on the liver and kidneys, by the method counteracts the reproduction of toxic products of protein breakdown [17]. The metabolic shift towards an increase in the acidity of the flora of the gastrointestinal tract not only reduces the frequency of reproduction of ammonia from amino acids and urea, but also significantly reduces its absorption into the blood, through conversion into an ionized ammonium form, which is not able to penetrate through the intestinal mucosa, as well as the result of the synthesis of lactulose bacterial protein is a decrease in ammonia levels [11, 15].

Andrey Georgievich Khramtsov who is a well-known scientist in the field of technology for deep processing of dairy raw materials, academician of the Russian Academy of Agricultural Sciences and academician of the Russian Academy of Sciences was one of the founders of the technology for producing prebiotic No. 1 - lactulose and was awarded the Prize of the Government of the Russian Federation for this development result.

Building on the previously published positive effects of Lactulose as a prebiotic, it promotes absorption and retention of calcium, magnesium, zinc and iron in the body, influences on indicators of body weight gain, stimulates the body's immune responses, and also has a beneficial effect on intestinal morphology, selectively stimulating growth beneficial to the health of bacteria. The present study was carried out to develop schemes for the complex interaction of lactulose, as a catalytic enzyme capable of selectively stimulating the growth of a given selected strain Lactobacillus acidophilus (B-4107) K-1-T with the prospect of using it for the prevention of gastrointestinal diseases in calves.

\section{Materials and methods of the studies}

On the basis of the scientific testing laboratory of the Department of Epizootology and Microbiology of the Faculty of Veterinary Medicine of the Federal State Budgetary Educational Institution of Higher Education "Stavropol State Agrarian University", in the period from 2020 to 2021 basic research was carried out to optimize the growth processes of Lactobacillus acidophilus with the prospect of its further use for the prevention of gastrointestinal diseases in calves.

The objects of research were the selected strain Lactobacillus acidophilus (B-4107) K1-T, acquired in the national bioresource center - the All-Russian collection of industrial microorganisms (NBC VKPM), characterized by antagonistic activity in relation to putrefactive and pyogenic bacteria; as well as the prebiotic Lactulose.

Microbiological studies were carried out in a laminar box, considering the rules of asepsis, for the cultivation of lactic acid microorganisms, the MRS medium (agar) was used at a temperature of $37^{\circ} \mathrm{C}$ for $24-48$ hours, the growing colonies were counted according to GOST 10444.11-2013 and GOST R 56139-2014.

Prebiotic Lactulose was added to $1 \mathrm{ml}$ of Lactobacillus acidophilus (B-4107) K-1-T (10 $\mathrm{CFU} / \mathrm{ml}$ ) in an amount of $0.02 \mathrm{~g} ; 0.05 \mathrm{~g} ; 0.08 \mathrm{~g} ; 0.11 \mathrm{~g} ; 0.15 \mathrm{~g}$ - respectively Experiment 1 , Experiment 2, Experiment 3, Experiment 4, Experiment 5. In the Control remained the 
probiotic Lactobacillus acidophilus (B-4107) K-1-T (10 $\mathrm{CFU} / \mathrm{ml})$ without the addition of lactulose. Dilutions of the components were carried out by adding saline 1: 1 and 1:2, as a result of which the number of column-forming units of the probiotic Lactobacillus acidophilus (B-4107) K-1-T and the amount of lactulose decreased, but the ratio of probiotic and prebiotic remained the same. After inoculation of $0.1 \mathrm{ml}$ of the components of the experiment and control on the MRS nutrient medium (agar) and cultivation, the average count of the grown colonies was carried out on 2 Petri dishes. The results of the experiments are reflected in the following section.

\section{Results}

Selected strain Lactobacillus acidophilus (B-4107) K-1-T is a freeze-dried culture of lactic acid microorganism in the amount of $10^{8} \mathrm{CFU} / \mathrm{ml}$. The species of Lactobacillus acidophilus is based on morphological, cultural and biochemical properties. When cultured on MRS (agar) medium, small, slightly convex, colorless colonies were formed. When stained by the Gram method, rod-shaped gram-positive, non-spore-forming microorganisms are visible. Biochemical properties are determined on the media of Giss - "Color row" (media with carbohydrates) (Table 1).

Table 1. Biochemical properties of strain Lactobacillus acidophilus (B-4107) K-1-T.

\begin{tabular}{|c|c|c|c|c|c|c|c|c|c|c|}
\hline \multirow[b]{2}{*}{ Strain name } & \multicolumn{10}{|c|}{ Carbohydrates } \\
\hline & $\begin{array}{l}0 \\
\ddot{O} \\
0 \\
0 \\
\end{array}$ & 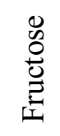 & $\begin{array}{l}0 \\
0 \\
0 \\
0 \overrightarrow{1}\end{array}$ & $\begin{array}{l}0 \\
0 \\
0 \\
0 \\
\frac{\pi}{0} \\
\tilde{J} \\
\tilde{J}\end{array}$ & 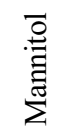 & 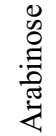 & 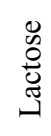 & $\frac{0}{0}$ & 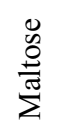 & $\begin{array}{l}0 \\
\ddot{0} \\
0 \\
0 \\
\tilde{D}\end{array}$ \\
\hline $\begin{array}{c}\text { Lactobacillus } \\
\text { acidophilus } \\
\text { (B-4107) K-1-T }\end{array}$ & + & + & - & + & + & - & + & - & + & + \\
\hline
\end{tabular}

According to the data of Table 1, it can be seen that Lactobacillus acidophilus (B-4107) $\mathrm{K}-1-\mathrm{T}$ on Giss media actively ferments glucose, fructose, mannose, galactose with the formation of acid and gas, and disaccharides, which include lactose, maltose, sucrose; does not ferment arabinose and xylose.

To determine the effect of the prebiotic Lactulose, as a catalytic enzyme, on the growth processes of the selected strain Lactobacillus acidophilus (B-4107) K-1-T, a laboratory experiment was carried out with various dosages of Lactulose. The dose of Lactulose addition ranged from 0.02 to $0.15 \mathrm{~g} / \mathrm{ml}$ to the diluted selected Lactobacillus acidophilus (B4107) $\mathrm{K}-1-\mathrm{T}$ strain $\left(10^{8} \mathrm{CFU} / \mathrm{ml}\right)$. After a 30-minute exposure, the components were inoculated onto the MRS nutrient medium (Table 2).

Table 2. Lactulose and Lactobacillus acidophilus (B-4107) K-1-T collection strain quantity.

\begin{tabular}{|c|c|c|c|c|c|c|}
\hline $\begin{array}{c}\text { Component } \\
\text { names }\end{array}$ & $\begin{array}{c}\text { Contro } \\
\mathbf{l}\end{array}$ & $\begin{array}{c}\text { Experime } \\
\text { nt 1 }\end{array}$ & $\begin{array}{c}\text { Experime } \\
\text { nt 2 }\end{array}$ & $\begin{array}{c}\text { Experime } \\
\text { nt 3 }\end{array}$ & $\begin{array}{c}\text { Experime } \\
\text { nt 4 4 }\end{array}$ & $\begin{array}{c}\text { Experi } \\
\text { ment 5 }\end{array}$ \\
\hline $\begin{array}{c}\text { Lactobacillus } \\
\text { acidophilus } \\
\text { (B-4107) } \\
\text { K-1-T, } \\
\text { CFU/ml }\end{array}$ & $10^{8}$ & $10^{8}$ & $10^{8}$ & $10^{8}$ & $10^{8}$ & $10^{8}$ \\
\hline Lactulose, g/ml & - & 0.02 & 0.05 & 0.08 & 0.11 & 0.15 \\
\hline
\end{tabular}


Microbiological studies to optimize the growth processes of the selected strain Lactobacillus acidophilus (B-4107) K-1-T were carried out in accordance with the generally accepted methods for calculating the total microbial count. From each test tube, $0.1 \mathrm{ml}$ was inoculated onto the surface of the selective agar MRS medium on 2 Petri dishes.

In parallel, inoculation was carried out from a control tube in which there was a selected strain of Lactobacillus acidophilus (B-4107) K-1-T $\left(10^{8} \mathrm{CFU} / \mathrm{ml}\right)$. Petri dishes with inoculations were incubated in a thermostat at $37{ }^{\circ} \mathrm{C}$ for $24-48$ hours, after which the number of colony-forming units was counted on MRS nutrient medium. The results of the experiment on optimizing the growth processes of the selected strain Lactobacillus acidophilus (B-4107) K-1-T are shown in the table (Table 3).

Table 3. Results of the experiment on optimization of the growth processes of the collection strain Lactobacillus acidophilus (B-4107) K-1-T.

\begin{tabular}{|c|c|c|c|c|}
\hline \multirow{2}{*}{ № } & \multirow{2}{*}{ Experiment } & $\begin{array}{c}\mathbf{1 0}^{\mathbf{8}} \\
\text { Lactobacillus } \\
\text { acidophilus } \\
\text { CFU/ml }\end{array}$ & $\begin{array}{c}\text { Lactobacillus } \\
\text { acidophilus } \\
\text { CFU/ml }\end{array}$ & $\begin{array}{c}\mathbf{1 0}^{\mathbf{4}} \\
\text { Lactobacillus } \\
\text { acidophilus } \\
\text { CFU/ml }\end{array}$ \\
\cline { 3 - 5 } & Experiment 1 & Intensive growth & Intensive growth & 4.5 \\
\hline 2. & Experiment 2 & Intensive growth & Intensive growth & 5.0 \\
\hline 3. & Experiment 3 & Intensive growth & Intensive growth & Intensive growth \\
\hline 4. & Experiment 4 & Intensive growth & Intensive growth & Intensive growth \\
\hline 5. & Experiment 5 & Intensive growth & Intensive growth & Intensive growth \\
\hline 6. & Control & Intensive growth & Intensive growth & 3.5 \\
\hline
\end{tabular}

In the course of the conducted microbiological studies, it was determined that in experiments with the addition of Lactulose, a more intensive growth of the selected strain Lactobacillus acidophilus (B-4107) K-1-T was observed, compared to the Control, which was observed in the dilution of $10^{2}$ Lactobacillus acidophilus. Intensive growth of the selected strain Lactobacillus acidophilus (B-4107) K-1-T was observed in experiments 1 and 2 with the optimal amount of the prebiotic Lactulose $0.02-0.05 \mathrm{~g} / \mathrm{ml}$. The growth rate of the selected strain Lactobacillus acidophilus (B-4107) $\mathrm{K}-1-\mathrm{T}\left(10^{8} \mathrm{CFU} / \mathrm{ml}\right)$, in comparison with the Control, increases with the addition of lactulose $0.02 \mathrm{~g} / \mathrm{ml}$ by $28.6 \%$, and with the addition of lactulose $0.05 \mathrm{~g} / \mathrm{ml}$ - by $42.9 \%$.

\section{Conclusions}

It was established that the prebiotic Lactulose, as a catalytic enzyme, has a significant effect on the growth and activity of the selected strain Lactobacillus acidophilus (B-4107) K-1-T. The growth rate of the selected strain Lactobacillus acidophilus (B-4107) K-1-T (10 ${ }^{8}$ $\mathrm{CFU} / \mathrm{ml}$ ) increases with the addition of lactulose $0.02 \mathrm{~g} / \mathrm{ml}$ by $28.6 \%$, and with the addition of lactulose $0.05 \mathrm{~g} / \mathrm{ml}$ - by $42.9 \%$. The use of lactulose in the treatment and prevention of gastrointestinal dysbiosis of ruminants is poorly developed. The results obtained will make 
it possible to develop effective means of normalizing the microbiota in the prevention of diseases of the gastrointestinal tract of infectious and non-infectious etiology in young cattle in postnatal ontogenesis.

\section{References}

1. S.-F. Li, F.-R. Zhao, J.-J. Shao, et al., International Immunopharmacology, 52, 253-260 (2017), doi: 10.1016/j.intimp.2017.08.028

2. C. Hill, F. Guarner, G. Reid, et al., Nature Rev Gastroenterol Hepatol, (11), 506-514 (2014), doi: 10.1038/nrgastro. 2014.662.

3. J. G. LeBlanc, C. Milani, Curr. Opin. Biotechnol., 24 (2), 160-168 (2013), doi: 10.1016/j.copbio.2012.08.005

4. T. Didari, S. Solki, S. Mozaffari, et al., Expert Opin. Drug Saf., 13(2), 227-239 (2014), doi: $10.1517 / 14740338.2014 .872627$

5. C. M. Carey, M. Kostrzynska, Can. J. Microbiol., 59(1), 9-17 (2013), doi: 10.1139/cjm-2012-0446

6. A. Duse, K. P. Waller, Journal of Dairy Science, 98 (9), 6387-6398 (2015), doi: 10.3168/jds.2014-8432

7. M. Wiseman, Dalhousie University (2012), doi: 10.21608/bvmj.2015.32504

8. G.B. Kim, Y.M. Seo, C.H. Kim, I.K. Paik, Poultry Science, 90(1), 75-82 (2011), doi: 10.3382/ps.2010-00732

9. E. Ohimain, R. Ofongo, International Journal of Animal and Veterinary Advances, 4(2), 135-143 (2012), doi: 10.1016/j.aninu.2018.08.002

10. A.A. Guerra-Ordaz, F. Molist, R.G. Hermes, et al., Anim., 185, 160-168 (2014), doi: 10.1128/AEM.00770-14

11. I. A. Naqid, J. P. Owen, B. Maddison, et al., Animal Feed Science and Technology, 201(9), 57-65 (2015), doi: 10.1016/j.anifeedsci.2014.12.005

12. L. Huiying, F. Breidt, J. Food Prot., 74 (6), 893-898 (2011), doi: 10.4315/0362028X.JFP-10-404

13. J. Scaria, L. D. Warnick, J. B. Kaneene, et al., Molecular and Cellular Probes, 24 (6), 325-45 (2010), doi: 10.1016/j.mcp.2010.07.004

14. N. Malmuthuge, Y. Chen, G. Liang, American Dairy Science Association, 98 (11), 8044-8053 (2015), doi: 10.3168 / jds.2015-9607

15. P. Hemarajata, J. Versalovic, Therapeutic Advances in Gastroenterology, 6 (1), 39-51 (2013), doi: 10.1177/1756283X12459294

16. L. Baffoni, V. Stenico, E. Strahsburger, et al., BMC Microbiology, 13, 149 (2013), doi: 10.1186/1471-2180-13-149

17. A. Sahagun-Ruiz, L. V. Velazquez, S. Bhaskaran, et al., Vet. Res. Commun., 39 (4), 229-236 (2015), doi: 10.1007/s11259-015-9646-1 